\title{
Caspase-cleavage of tau is an early event in Alzheimer disease tangle pathology
}

\author{
Robert A. Rissman, ${ }^{1}$ Wayne W. Poon, ${ }^{1}$ Mathew Blurton-Jones, ${ }^{1}$ Salvatore Oddo, ${ }^{1}$ Reidun Torp, ${ }^{1}$ \\ Michael P. Vitek, ${ }^{2}$ Frank M. LaFerla, ${ }^{1}$ Troy T. Rohn, ${ }^{3}$ and Carl W. Cotman' ${ }^{1}$
}

\begin{abstract}
${ }^{1}$ Institute for Brain Aging and Dementia, University of California, Irvine, California, USA. 2Department of Neurology, Duke University Medical Center, Durham, North Carolina, USA. ${ }^{3}$ Department of Biology, Boise State University, Boise, Idaho, USA.
\end{abstract}

\begin{abstract}
Neurofibrillary tangles (NFTs) are composed of abnormal aggregates of the cytoskeletal protein tau. Together with amyloid $\beta(A \beta)$ plaques and neuronal and synaptic loss, NFTs constitute the primary pathological hallmarks of Alzheimer disease (AD). Recent evidence also suggests that caspases are activated early in the progression of AD and may play a role in neuronal loss and NFT pathology. Here we demonstrate that tau is cleaved at D421 ( $\Delta$ Tau) by executioner caspases. Following caspase-cleavage, $\Delta$ Tau facilitates nucleation-dependent filament formation and readily adopts a conformational change recognized by the early pathological tau marker MC1. $\triangle$ Tau can be phosphorylated by glycogen synthase kinase- $3 \beta$ and subsequently recognized by the NFT antibody PHF-1. In transgenic mice and AD brains, $\triangle$ Tau associates with both early and late markers of NFTs and is correlated with cognitive decline. Additionally, $\Delta$ Tau colocalizes with $A \beta_{1-42}$ and is induced by $A \beta_{1-42}$ in vitro. Collectively, our data imply that $A \beta$ accumulation triggers caspase activation, leading to caspase-cleavage of tau, and that this is an early event that may precede hyperphosphorylation in the evolution of AD tangle pathology. These results suggest that therapeutics aimed at inhibiting tau caspase-cleavage may prove beneficial not only in preventing NFT formation, but also in slowing cognitive decline.
\end{abstract}

\section{Introduction}

Tau is a microtubule-associated protein that stabilizes the neuronal cytoskeleton and participates in vesicular transport and axonal polarity (1-4). In the brain, there are six isoforms of tau, produced by alternative mRNA splicing of a single gene located on chromosome 17. Pathological alterations in tau occur in several neurodegenerative disorders, including Alzheimer disease (AD), supranuclear palsy, and frontotemporal dementia with parkinsonism (5).

In $\mathrm{AD}$, insoluble neurofibrillary tangles (NFTs) composed of hyperphosphorylated forms of tau accumulate initially within the entorhinal cortex and CA1 subfield of the hippocampus (6-8). Recent studies have begun to clarify the sequence of tau alterations that lead to neurodegeneration, including conformational changes and hyperphosphorylation. Antibodies developed against pathological tau have helped to identify these changes. An aberrant folded conformational change in tau, recognized with antibody MC1, appears to be one of the earliest tau pathological events (9-12). The antibody AT8 recognizes tau phosphorylated at both serine 202 and threonine 205, which are the first residues to be hyperphosphorylated, whereas the antibody PHF-1 recognizes phosphorylation at serines 396 and 404 and reacts with more mature hyperphosphorylated forms of tau found primarily within late-stage tangles (13-15). Such alterations in tau may reduce its binding affinity for microtubules, thereby leading to depolymerization of microtubules and contributing to the neuronal loss observed in AD (16-18).

Nonstandard abbreviations used: Alzheimer disease (AD); amyloid $\beta(A \beta)$; glycogen synthase kinase-3 $\beta$ (GSK-3 $\beta$ ); laser light scattering (LLS); mild cognitive impairment (MCI); Mini-Mental State Examination (MMSE); neurofibrillary tangle (NFT); paired helical filament (PHF); radioimmunoprecipitation buffer (RIPA); reassembly buffer (RAB); traumatic brain injury (TBI); triple-transgenic mouse model (3xTg-AD).

Conflict of interest: The authors have declared that no conflict of interest exists.

Citation for this article: J. Clin. Invest. 114:121-130 (2004).

doi:10.1172/JCI200420640.
Caspases are cysteine aspartate proteases that are critically involved in apoptosis. These enzymes can be broadly divided into initiator and executioner caspases, with the former functioning to initiate apoptosis by activating executioner caspases and the latter acting on downstream effector substrates that result in the progression of apoptosis and the appearance of hallmark morphological changes such as cell shrinkage, nuclear fragmentation, and membrane blebbing (19). Increasing evidence suggests that caspases are activated in the $\mathrm{AD}$ brain (20-26). Furthermore, components of the neuronal cytoskeleton, including tau, are targeted by caspases following apoptotic stimuli $(23,27-34)$. Although the role of tau caspase-cleavage in $\mathrm{AD}$ pathology remains unresolved, recent evidence now implicates the caspase-cleavage of tau in tangle pathology (34). However, in $\mathrm{AD}$, it is unclear whether hallmark pathological lesions induce caspases, whether caspases contribute to these lesions, or whether both occur.

In this study, we tested the hypothesis that caspase-cleavage of tau is an early event in tangle formation in both $\mathrm{AD}$ and a transgenic model of this disorder. We found that caspase-cleaved tau catalyzes filament formation, adopts a conformation found in early-stage tangles, and can be hyperphosphorylated. Caspasecleavage of tau also colocalizes with amyloid $\beta(A \beta)$ and developing tangles in both transgenic mice and the $\mathrm{AD}$ brain. In primary cortical neurons, $A \beta$-induced caspase activation leads to tau cleavage and generates tangle-like morphology. Thus, our data suggest that caspase activation is an early event in NFT formation that can be triggered by $A \beta$, and that caspase activation may contribute to an important hallmark lesion of $\mathrm{AD}$.

\section{Results}

Tau is cleaved at D421 by executioner caspases. To determine which caspases cleave tau, recombinant human tau 40 was treated with several active caspases. Incubation of tau with caspase- 3 or -7 , but not with caspase- $1,-4,-5,-8$, or -10 , resulted in a $2-\mathrm{kDa}$ electrophoretic shift by SDS-PAGE (Figure 1A, arrowhead). Analysis of 
A
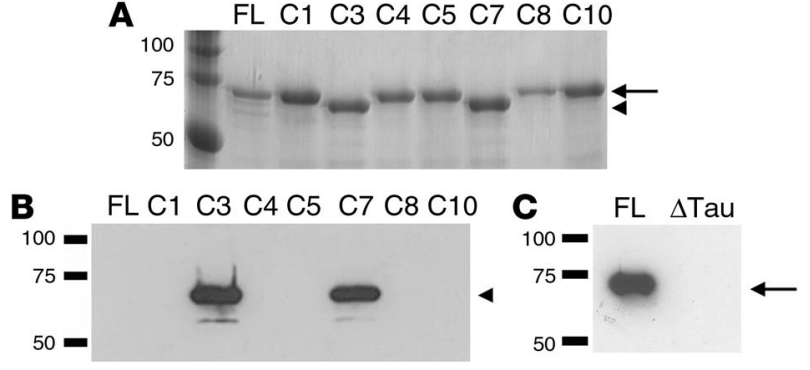

Figure 1

Executioner caspases cleave tau at D421. (A) Recombinant human tau40 (Calbiochem) treated with various caspases was separated by SDS-PAGE and stained with Coomassie blue. Treatment with caspase-3 (C3) and caspase-7 (C7) resulted in a 2-kDa shift (arrowhead) relative to full-length tau $(\mathrm{FL})$, while treatment with caspase-1 (C1), -4 (C4), -5 (C5), -8 (C8), or -10 (C10) did not (arrow). (B) Western blot analysis confirmed the specificity of polyclonal antibody $(\alpha-\Delta T a u)$ to D421 caspase-cleaved tau. $\alpha-\Delta$ Tau recognized caspase-3- and caspase-7-cleaved tau, but not uncleaved tau. (C) Executioner caspases remove the C-terminal epitope of tau that is recognized by antibody T46. Full-length and caspase-cleaved tau was probed with mAb T46.

the cleavage products by electrospray mass spectroscopy revealed two tau fragments with molecular weights of $45,900.00 \mathrm{Da}(\Delta \mathrm{Tau})$ and 1,997.99 $\mathrm{Da}$ (C-terminus), corresponding to the theoretical molecular weights of tau cleaved after amino acid residue D421 $(45,900.80 \mathrm{Da}$ and $1,999.29 \mathrm{Da})$ (see supplemental data; available at http://www.jci.org/cgi/content/full/114/1/121/DC1). Cleavage after $\mathrm{D} 421$ was also confirmed by primary amino acid sequencing of the small fragment (data not shown).

To examine the role of $\Delta$ Tau in NFT pathology, we generated a rabbit polyclonal antibody $(\alpha-\Delta \mathrm{Tau})$ directed against the caspasecleaved carboxy-terminus of tau. The specificity of $\alpha-\Delta$ Tau was confirmed by Western blot analysis and was found to selectively recognize the D421-cleaved fragment generated by caspase-3 or -7 but not full-length tau (Figure 1B). In contrast, a previously generated antibody that recognizes the C-terminus of tau (T46; ref. 35, 36) detects full-length tau but not $\Delta$ Tau (Figure 1C).

Specificity of the $\alpha-\Delta$ Tau antibody was further assessed, in vivo, using traumatic brain injury (TBI), a model that leads to neuronal caspase activation (37). Forty-eight hours after injury, immunohistochemical analysis confirmed caspase-3 activation within both wild-type (Figure 2A, inset) and $\mathrm{Tan}^{-/}$brains (Figure $2 \mathrm{~B}$, inset). However, $\Delta$ Tau immunoreactivity was observed within wild-type (Figure 2A) but not Tan ${ }^{-/}$(Figure 2B) mice following TBI. In contrast, caspase-cleaved fodrin, another cytoskeletal target of caspases, was observed within both wild-type and tau knockout brains (see supplemental data). Therefore, the $\alpha-\Delta$ Tau antibody specifically detects caspase-cleaved tau and does not cross-react with other caspase-cleaved substrates in vivo.

$\Delta$ Tau is prevalent within the $A D$ brain and is inversely correlated with cognitive function. To determine whether $\Delta \mathrm{Tau}$ is associated with $\mathrm{AD}$ neuropathology, $\mathrm{AD}$ and control hippocampal sections were immunolabeled with the $\alpha-\Delta$ Tau antibody. We observed extensive $\alpha-\Delta$ Tau labeling within AD hippocampus (Figure $3 \mathrm{~A}$ ). In contrast, only occasional $\Delta$ Tau-positive cells were observed in controls (Figure 3B). Preadsorption of the $\alpha-\Delta$ Tau antibody with immunizing peptide led to a loss of all immunoreactivity (Figure 3A, inset).
Next, we performed light-level immunohistochemistry to quantify the number of $\Delta$ Tau-immunoreactive cells within CA1 of $\mathrm{AD}$ versus control hippocampus (data not shown). Significantly more $\Delta$ Tau-immunoreactive cells were observed in AD than in control brains (AD, $28.2 \pm 1.65$; control, $4.25 \pm 0.85 ; P<0.05$ ). Furthermore, the number of $\Delta$ Tau-immunoreactive cells within CA1 of $\mathrm{AD}$ cases inversely correlated with cognitive function (Mini-Mental State Examination [MMSE] score) (Figure 3C). Thus, $\Delta$ Tau may contribute to cognitive decline in $\mathrm{AD}$.

To assess the relationship between caspase-cleaved tau and fulllength tau pathology, fluorescent double labeling for $\Delta$ Tau and the C-terminal-specific antibody T46 was performed (36). High-power confocal imaging revealed an intriguing difference in subcellular distribution between these two markers, suggesting that both fulllength tau (T46, green) and cleaved tau ( $\alpha-\Delta$ Tau, red) are present within the same NFTs (Figure 3, D and E). T46 immunoreactivity was frequently observed surrounding $\Delta$ Tau immunofluorescence in both cell bodies (Figure 3D) and dystrophic neurites (Figure 3E). The C-terminal epitope recognized by T46 (35) is liberated by executioner caspases (Figure 1C). Therefore, the minimal subcellular colocalization of $\Delta$ Tau and T46 further confirms the cleavage site specificity of $\alpha-\Delta$ Tau.

Tau becomes increasingly insoluble as $\mathrm{AD}$ progresses. Therefore, we performed sequential fractionation of control, mild cognitive impairment (MCI), and $\mathrm{AD}$ brain lysates into high-salt reassembly buffer (RAB) and detergent-soluble radioimmunoprecipitation buffer (RIPA) fractions to examine the solubility of $\Delta$ Tau as $\mathrm{AD}$ progresses. We found that $\Delta$ Tau recognized multiple caspase-cleaved tau isoforms in both MCI and $\mathrm{AD}$ cases but not controls within the high-salt soluble RAB fraction (Figure 4A). In the detergent-soluble RIPA fraction, $\Delta$ Tau was present within $\mathrm{AD}$ cases and also in a more advanced MCI-AD transitional case (Figure 4A, asterisk), but not within MCI or control cases (Figure 4A). In addition, $\Delta$ Tau appeared to be post-transationally modified (hyperphosphorylation or ubiquitination) within the RIPA fraction, as evidenced by smeared signal (Figure 4A). Therefore, $\Delta$ Tau appears to become increasingly insoluble as $\mathrm{AD}$ advances.
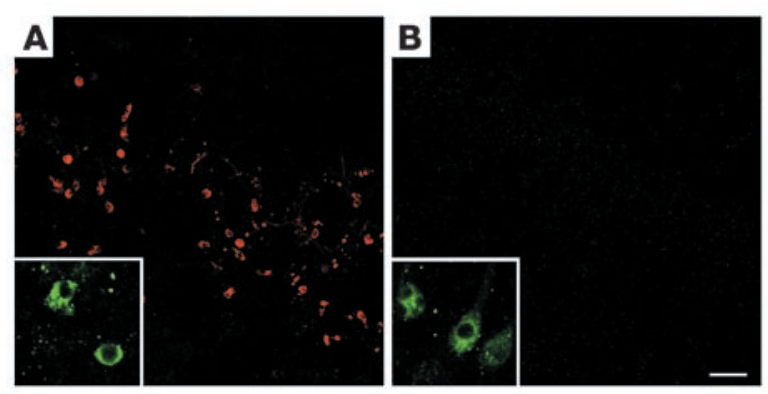

\section{Figure 2}

The $\alpha-\Delta$ Tau antibody specifically recognizes caspase-cleaved tau in vivo. Wild-type (A) and $\mathrm{Tau}^{-1}$ (B) mice were subjected to TBI to induce caspase activation. Immunofluorescent labeling with an antibody to active caspase-3 confirmed caspase activation within both wild-type (A, inset) and tau knockout (B, inset) brains. $\Delta$ Tau immunoreactivity was also detected within wild-type brains (A). In contrast, $\Delta$ Tau immunoreactivity was not observed within tau knockout mice following TBI (B), which further confirms the specificity of the $\alpha-\Delta$ Tau antibody in vivo. Scale bar: $80 \mu \mathrm{m}$ (A and B), $20 \mu \mathrm{m}$ (insets). 

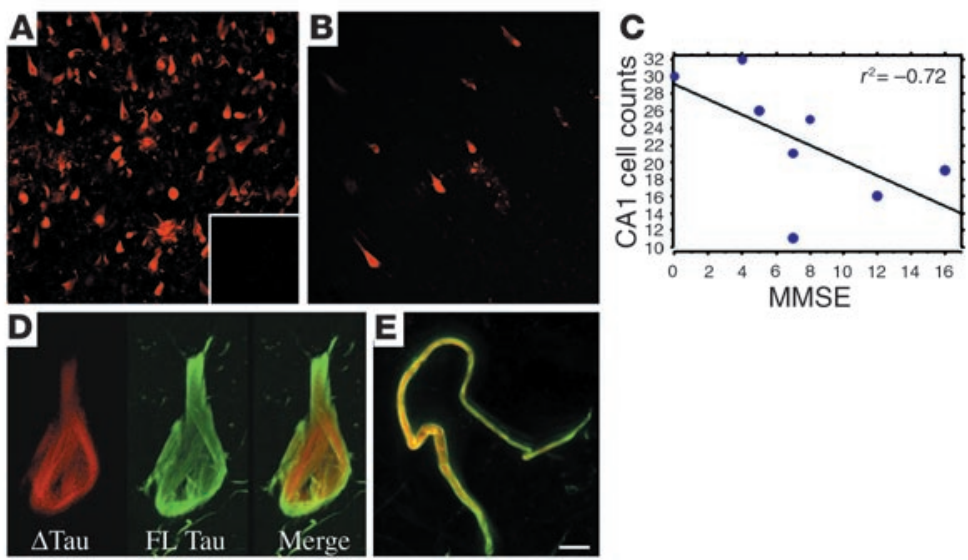

Figure 3

$\Delta T a u$ is found predominantly within $A D$ brain and is inversely correlated with cognitive function. Large numbers of $\Delta T a u$-immunoreactive neurons (red) were observed in the hippocampus and entorhinal cortex of AD brains (A). In contrast, only occasional $\Delta$ Tau-immunoreactive cells were observed in age-matched control cases (B). Preadsorption of the $\alpha-\Delta$ Tau antibody with immunizing peptide resulted in no immunofluorescence (A, inset). Regression analysis of the number of hippocampal CA1 cells labeled with $\alpha-\Delta$ Tau versus AD Mini-Mental State Examination (MMSE) scores revealed a significant inverse correlation between $\Delta$ Tau and cognitive function $\left(P=0.05, r^{2}=-0.72\right.$; (C). High-power confocal microscopy demonstrated distinct subcellular localization of $\Delta T a u$ (red) and the C-terminal-specific antibody T46 (green) within cell bodies (D) and dystrophic neurites (E). Scale bar: $65 \mu \mathrm{m}$ (A and B), $8 \mu \mathrm{m}$ (D), $5 \mu \mathrm{m}$ (E).

To further elucidate the subcellular distribution of $\Delta$ Tau in the $\mathrm{AD}$ brain, immunogold electron microscopy was performed using the $\alpha-\Delta$ Tau antibody. A diffuse distribution of $\alpha-\Delta$ Tau conjugated gold particles was observed in association with tangle-like structures within neuronal somata (Figure 4B). In addition, gold particles were observed within structurally intact axons (Figure 4C), which indicates that caspase-cleavage of tau may precede neuritic pathology.

$\Delta$ Tau induces filament formation. To determine whether $\Delta$ Tau contributes to tangle pathology by promoting filament formation, we measured light scattering, which has previously been shown to be an effective means of monitoring tau filamentous aggregates (38). Tau was incubated in the presence of heparin, which accelerates tau aggregation in vitro, so that we could assay filament formation in real time (39). We found that caspase-3-cleaved tau (filled squares) demonstrated increased light scattering over time (indicative of filament formation) and aggregated more rapidly than full-length tau (filled diamonds, Figure 5). In addition, $\Delta$ Tau accelerated filament formation of full-length tau, as the incubation of full-length tau in the presence of $\Delta$ Tau resulted in a dramatic increase in the rate of scattered light (open squares, Figure 5). These results show not only that caspase-cleavage of tau leads to increased filament formation, but that $\Delta$ Tau may also act to seed filament formation of full-length tau. These data further support a nucleation-dependent mechanism of tau filament assembly (40).

Caspase-cleavage induces a conformational change in tau, which can be byperphosphorylated by glycogen synthase kinase- $3 \beta$. The increased propensity of $\Delta$ Tau to induce filament formation may ensue from a conformational change that follows liberation of the C-terminus. One of the earliest pathological alterations in tau during $\mathrm{AD}$ is a conformational change that is recognized by the antibody $\mathrm{MC} 1$ (12). Because the formation of the MC1 epitope occurs prior to paired helical filament (PHF) assembly (12), we hypothesized that $\Delta$ Tau may likewise adopt this conformation. Immunoprecipitation with MC1 preferentially recognized $\Delta$ Tau over full-length tau in three separate experiments (Figure 6A). Therefore, our results show that caspase-cleavage of tau induces an early conformational change recognized by MC1.

Besides a conformational change, phosphorylation of specific residues is another important stage in the pathology of tau. Accordingly, we tested whether caspase-cleaved tau can be hyperphosphorylated in vitro. Recombinant full-length tau and caspase-cleaved tau were incubated at $37^{\circ} \mathrm{C}$ in the presence of ATP and glycogen synthase kinase-3 $\beta$ (GSK-3 $\beta$ ), a kinase implicated in the hyperphosphorylation of tau (41). Western blot analysis with the tau phospho-epitope antibody PHF-1 revealed that both $\Delta$ Tau and full-length tau were hyperphosphorylated in vitro by GSK-3 $\beta$ (Figure 6B). Although we assayed kinase activity over a 4-hour period, phosphorylation of $\Delta$ Tau occurred rapidly within 1 hour. Therefore, caspase-cleavage does not preclude subsequent hyperphosphorylation of tau, which further supports the notion that $\Delta$ Tau may occur early in the development of NFT pathology.

$\triangle T a u$ is observed throughout the evolution of NFT pathology in the $A D$ brain. Because $\triangle T$ Tau adopts a conformation that is recognized by the MC1 antibody and can be phosphorylated to form the PHF-1 epitope, we performed triple-labeling experiments with $\alpha-\Delta$ Tau (red), MC1 (green), and fluorescently labeled PHF-1 (blue) to determine whether $\Delta$ Tau colocalized with early (MC1) or late (PHF-1) tau pathological alterations in $\mathrm{AD}$ (Figure 7). In the $\mathrm{AD}$ hippocampus and entorhinal cortex, we found both neurons (Figure 7, A-D) and dystrophic neurites (Figure 7, E and F) that labeled with $\Delta$ Tau, $\mathrm{MC1}$, and PHF-1. We observed all three labels in pretangle (Figure $7 \mathrm{~A}$ ), diffuse tangle-bearing (Figure 7B), and mature tangle-bearing neurons (Figure 7, C and D). Interestingly, we did not observe

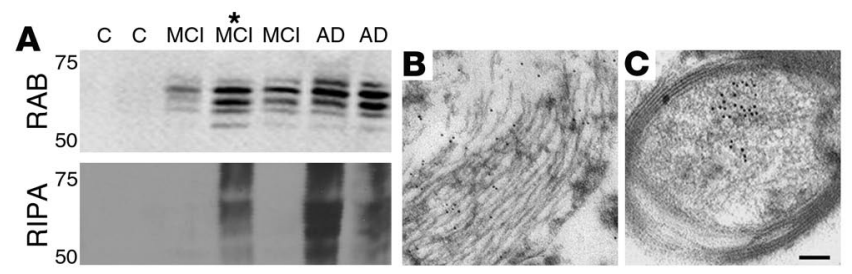

\section{Figure 4}

$\Delta$ Tau becomes increasingly insoluble with disease progression and is associated with tangle-like pathology at the ultrastructural level. Control (C), $\mathrm{MCl}$, and $\mathrm{AD}$ temporal cortex samples were subjected to sequential fractionation into high-salt RAB and detergent-soluble RIPA fractions as previously described $(50,78)(\mathbf{A})$. $\Delta$ Tau was detected in the soluble $\mathrm{RAB}$ fraction of $\mathrm{MCl}$ and $\mathrm{AD}$ but not control cases, suggesting that $\triangle \mathrm{Tau}$ production coincides with the early stages of cognitive decline in $A D$. In detergent-soluble RIPA fractions, $\triangle$ Tau was only detected in higherpathology $\mathrm{MCl}$ and $\mathrm{AD}$ cases, which suggests that $\triangle \mathrm{Tau}$ becomes more insoluble as $A D$ progresses. *This patient was considered transitional between $\mathrm{MCl}$ and $\mathrm{AD}$ (see Methods). Immunogold electron microscopy demonstrated $\Delta \mathrm{Tau}$ at the ultrastructural level in association with early tangle-like pathology within a neuronal cell body (B) and a myelinated axon (C). Scale bar: $0.4 \mu \mathrm{m}$ (B), $0.3 \mu \mathrm{m}$ (C). 


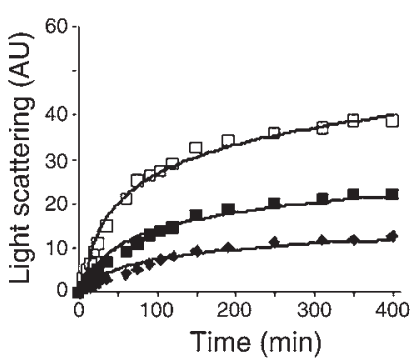

Figure 5

Caspase-cleavage of tau drives filament formation of full-length tau in vitro. Laser light scattering was used to assess tau filament formation in vitro. Equimolar $(4 \mu \mathrm{M})$ amounts of $\Delta T a u$ (filled squares), full-length tau (filled diamonds), or a 3:1 ratio of full-length tau to $\Delta T a u$ (open squares) were monitored over 400 minutes. $\Delta$ Tau (filled squares) scattered light (arbitrary units [AU]) more rapidly and to a greater degree than full-length tau (filled diamonds), indicating increased filament formation. Most interestingly, a 3:1 ratio of full-length tau to $\Delta$ Tau (open squares) dramatically accelerated light scattering, suggesting that $\Delta$ Tau may nucleate the assembly of full-length tau filaments.

any cells that expressed MC1 and PHF-1 without $\Delta$ Tau, which strengthens our assertion that $\Delta \mathrm{Tau}$ is important in tangle formation. Finally, triple labeling of plaque-associated dystrophic neurites revealed numerous permutations of the three tangle markers, suggesting that dystrophic neurites also show varying degrees of $\mathrm{AD}$ pathological progression (Figure 7F).

Active caspase-3 colocalizes with $\Delta$ Tau within pretangle neurons and dystrophic neurites of the $A D$ brain. To further examine whether caspase activation and caspase-cleavage of tau occur early in the formation of tangles, we performed fluorescent double labeling for active caspase- 3 and $\Delta \mathrm{Tau}$. We observed colocalization of active caspase-3 with $\Delta$ Tau, specifically within pretangle neurons of CA1 (Figure 8A) and dystrophic neurites (Figure 8B). In contrast, within mature extracellular tangles, active caspase- 3 was not observed, possibly because of loss of membrane integrity and cell death (Figure 8C). Taken together, these data suggest that caspase activation and caspase-cleavage of tau occur early in tangle development. However, caspase activation appears to be lost with cell death, while $\Delta$ Tau persists within extracellular NFTs, perhaps as insoluble accumulations.

Evidence that $\Delta$ Tau is induced by $A \beta . A \beta$ activates caspases in vitro (42-44). Therefore, we sought to examine whether $\Delta$ Tau colocalized with $\mathrm{A} \beta$ in the $\mathrm{AD}$ brain. We found that $\Delta \mathrm{Tau}$ (red) colocalized with granular intraneuronal $A \beta$ deposits (green) within CA1 (Figure 8, D and E). Similar results were observed with two different $A \beta$-specific antibodies $\left(A \beta_{1-42}\right.$ and $\left.4 \mathrm{G} 8\right)$. $\Delta$ Tauimmunoreactive dystrophic neurites were also frequently observed in association with $\mathrm{A} \beta$-immunoreactive plaques (Figure $8 \mathrm{~F}$ ). Combined, these data suggest that both intracellular and extracellular $\mathrm{A} \beta$ may induce caspase-cleavage of tau.

Because we found that $\mathrm{A} \beta$ colocalized with $\Delta \mathrm{Tau}$ in the $\mathrm{AD}$ brain, we determined whether $A \beta$ might induce caspase-cleavage of tau in cultured rat primary cortical neurons. Using immunofluorescent triple labeling for $\Delta$ Tau (red), the neuronal marker MAP-2 (green), and DAPI (blue), we found a significant increase in $\Delta$ Tau-positive neurons following fibrillar $A \beta_{1-42}$ treatment (Figure 9). Quantification of cell number (see Methods) revealed a significant increase in $\Delta$ Tau-positive cells following $\mathrm{A} \beta_{1-42}$ treatment $(F(7,31)=43.28$, $P<0.0001)$. After 6 hours of treatment, a twofold increase in $\Delta$ Tau- immunoreactive cells was observed (control, $1.0 \pm 0.33$ cells; treated, $2.13 \pm 0.35$ cells; $P=0.012$ ). However, by 24 hours, the number of $\Delta$ Tau-immunoreactive cells was increased almost ninefold over that of untreated cells (control, $0.75 \pm 0.37$ cells; treated, $6.63 \pm 0.38$ cells; $P<0.0001$ ) (Figure 9D). The number of $\Delta$ Tau-immunoreactive cells was not significantly affected after only 1 hour $(P=0.560)$ or 4 hours $(P=0.086)$ of $A \beta_{1-42}$ treatment. The majority of $\Delta$ Tau-bearing neurons $(91.5 \%)$ in control and $\mathrm{A} \beta_{1-42}$-treated cultures contained apoptotic nuclei, as assessed by DAPI (Figure 9, A-C, blue). Furthermore, we discovered that $\Delta$ Tau labeled striking tangle-like structures in dying neurons (Figure 9, B and C). Therefore, $A \beta_{1-42}$ triggers the production of $\Delta$ Tau in vitro.

$\Delta$ Tau is detected early in the progression of tangle pathology in a tripletransgenic model of $A D$. To better assess the role of caspase-cleaved $\Delta$ Tau in the pathogenesis of $A D$, we determined whether $\Delta$ Tau was present in the brains of transgenic mice. We used a recently characterized triple-transgenic mouse $(3 \times \mathrm{Tg}-\mathrm{AD})$ that recapitulates many salient features of $\mathrm{AD}$ : progressively developing intraneuronal $\mathrm{A} \beta$, deficits in long-term potentiation, amyloid plaques, and, subsequently, NFTs (45). We examined brains from 6- (with only A $\beta$ deposits but no NFT pathology), 12- (in the initial stages of NFT pathology), and 18-month-old mice (in which both plaque and NFT were well established). At 6 months of age, we detected intracellular $\mathrm{A} \beta$, but not $\Delta$ Tau immunoreactivity (data not shown). By 12 months of age, we found that $\Delta$ Tau (red) colocalized with intraneuronal $A \beta$ immunoreactivity within CA1 neurons of the hippocampus (Figure 10A). In older mice (18 months), $A \beta$ and $\Delta$ Tau colocalized within both CA1 neurons (Figure 10B) and the

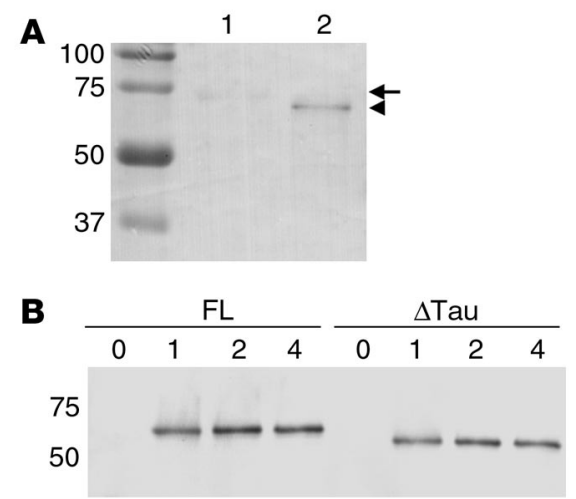

\section{Figure 6}

Caspase-cleavage of tau induces a conformational epitope that is recognized by the antibody MC1 and can be phosphorylated by GSK3- $\beta$. (A) MC1, a mAb that recognizes an early tau conformational change, was coupled to protein $G$ and used to immunoprecipitate either fulllength (lane 1) or caspase-3-cleaved recombinant tau (lane 2). Western blot analysis of immunoprecipitated proteins probed with an anti-tau polyclonal antibody revealed an increased affinity of the MC1 antibody for $\Delta$ Tau over full-length tau. (B) In vitro phosphorylation of $\Delta$ Tau: Either full-length or caspase-3-cleaved tau was phosphorylated with the kinase GSK-3 $\beta$ and then analyzed by Western blot with the phosphorylation-dependent antibody PHF-1. Immunoreactivity for PHF-1 demonstrates that, like full-length tau (FL), caspase-cleaved tau ( $\triangle \mathrm{Tau}$ ) can be hyperphosphorylated and suggests that caspasecleavage of tau does not preclude subsequent hyperphosphorylation. Numbers over each lane indicate the number of hours that tau species were incubated with GSK-3ß. MC1 and PHF-1 experiments were replicated three times, and representative immunoblots are shown. 

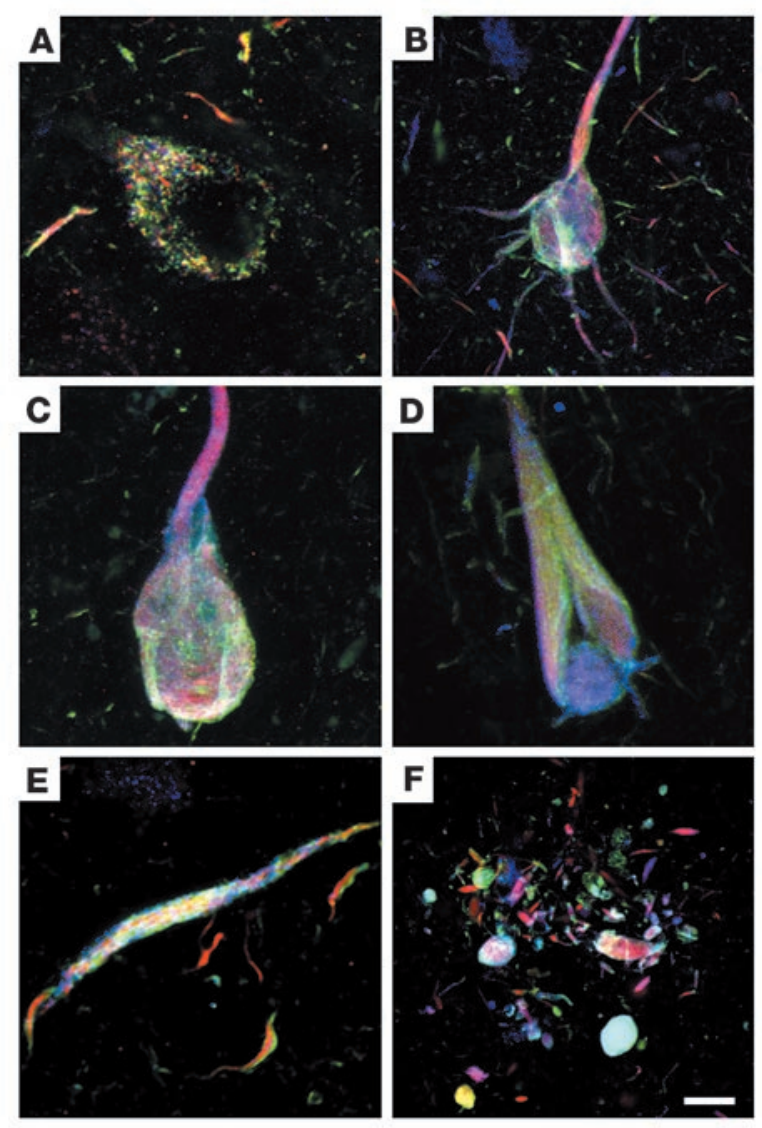

neocortex (Figure 10C). This finding closely mimics the progression of NFT pathology that has previously been reported in $3 \times \mathrm{Tg}$ $\mathrm{AD}$ mice (45). Next, we investigated whether $\Delta$ Tau colocalized with MC1 in this transgenic model. Colocalization of $\Delta \mathrm{Tau}$ (red) and MC1 (green) was first observed within CA1 pyramidal neurons at 12 months of age (Figure 10D) and persisted in 18-month-old mice (Figure 10, E and F). Interestingly, the subcellular distribution of $\Delta$ Tau evolved with increasing age. At 12 months, the great majority of $\Delta$ Tau-immunoreactive cells displayed punctate cleavage product within the soma and proximal dendrites of neurons (Figure 10, A and D). However, by 18 months of age, many $\Delta$ Tau-bearing neurons exhibited aggregated filamentous structures that appeared strikingly similar to AD tangle pathology (Figure 10, C and F).

\section{Figure 8}

Caspase activation and intracellular $A \beta$ colocalize with $\Delta \mathrm{Tau}$ in the $A D$ brain. Fluorescent confocal imaging demonstrated that $\Delta$ Tau (red) and active caspase-3 (green) colocalized within pretangle neurons (A) and dystrophic neurites (B) of the AD hippocampus. In contrast, mature extracellular tangles contained $\Delta$ Tau without evidence of caspase activation (C). Intracellular $A \beta$ (green), a known initiator of caspases, colocalizes with $\Delta T$ Tau (red) within AD neurons ( $D$ and E). In addition, $\Delta$ Tau-immunoreactive dystrophic neurites (red) were frequently associated with extracellular amyloid plaques (F). Scale bar: $5 \mu \mathrm{m}(\mathbf{A}), 1.5 \mu \mathrm{m}(\mathbf{B}), 6 \mu \mathrm{m}(\mathbf{C}), 3 \mu \mathrm{m}$ (D and E), $25 \mu \mathrm{m}(\mathbf{F})$.

\section{Figure 7}

$\Delta$ Tau is present throughout the evolution of NFT pathology. Triple immunofluorescence was used to examine the association of $\Delta T a u$ (red) with the conformation-sensitive tau antibody MC1 (green) and the tau phosphoepitope antibody PHF-1 (blue) in hippocampal NFT pathology. $\Delta \mathrm{Tau}$ (red) was coincident with $\mathrm{MC} 1$ and $\mathrm{PHF}-1$ throughout the evolution of NFT pathology within pretangle neurons (A), diffuse tangle-bearing neurons (B), mature tangle-bearing neurons ( $\mathbf{C}$ and D), and dystrophic neurites (E and F). Scale bar: $8 \mu \mathrm{m}(\mathbf{A}, \mathbf{C}$, and $\mathbf{D})$, $12 \mu \mathrm{m}$ (B), $4 \mu \mathrm{m}$ (E), $10 \mu \mathrm{m}$ (F).

\section{Discussion}

Here we demonstrate that caspase-cleavage of tau near the carboxy-terminus leads to a conformational change that is preferentially recognized by the early tangle marker MC1. $\Delta$ Tau also accelerates the aggregation of full-length tau filaments and can be hyperphosphorylated in vitro. Since $\Delta$ Tau colocalizes with MC1 within neurons of subjects with $\mathrm{AD}$ and $3 \mathrm{xTg}-\mathrm{AD}$ mice, we postulate that caspase-cleavage of tau occurs early in NFT formation. This hypothesis is further supported by the presence of $\Delta$ Tau in brain extracts from patients with MCI and the detection of $\Delta \mathrm{Tau}$ within pretangle-like structures by electron microscopy. However, the fact that $\Delta$ Tau colocalizes with PHF- 1 in the AD brain suggests that although $\Delta$ Tau may occur early in NFT formation, it persists throughout the evolution of NFTs.

The kinetics data support a role for $\Delta$ Tau in seeding tau pathology via a nucleation-dependent mechanism. Tau dimerization and subsequent nucleation events are proposed to be the rate-limiting step in filament formation (40). Because caspase-cleaved tau enhanced the rate of full-length tau filament formation, we speculate that caspase-cleavage of tau may either increase the propensity for tau to dimerize and/or affect nucleation-dependent filament assembly. Because MC1immunoreactive tau aggregates more readily than recombinant tau (12), the adopted MC1 conformation may explain why $\Delta$ Tau enhances filament formation. The MC1 conformation may involve the intramolecular interaction between the $\mathrm{N}$ - and C-termini of tau (folded conformation). Caspase-cleavage of tau may initiate the MC1 conformation by removing a portion of the C-terminus that inhibits tau aggregation in vitro (46), and allowing tau to adopt this conformation.
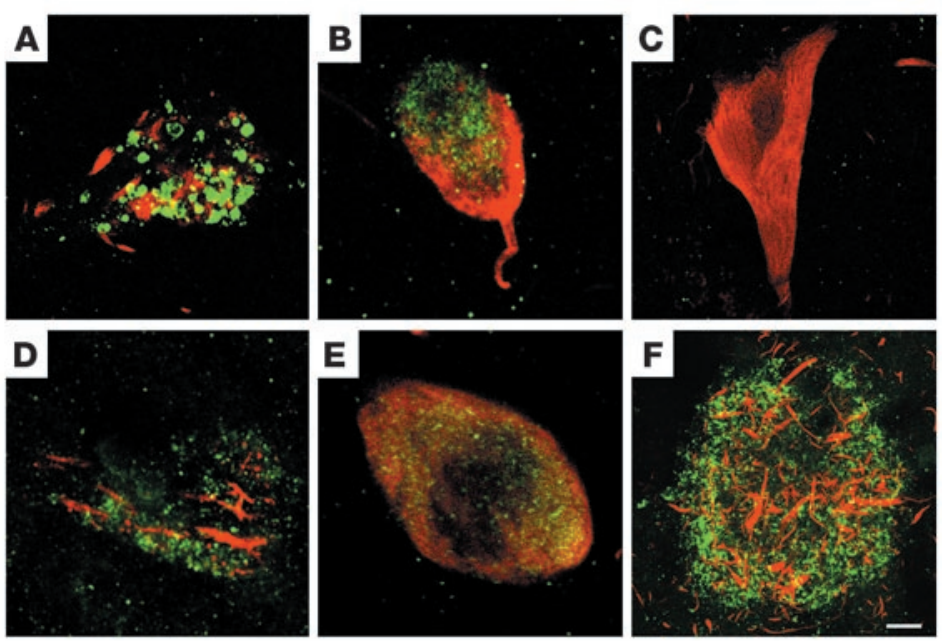

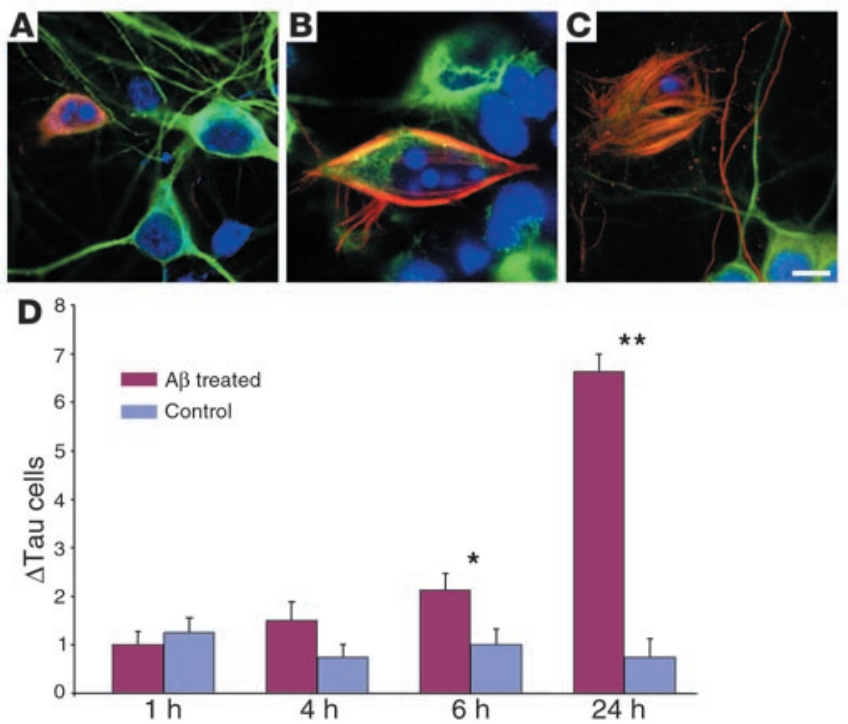

Figure 9

Treatment of cortical neurons with $A \beta$ leads to increased $\Delta$ Tau immunoreactivity. Fluorescent triple labeling of rat primary cortical neurons for $\triangle T a u$ (red), MAP-2 (green), and DAPI (blue) following $A \beta$ treatment $(\mathbf{A}-\mathbf{C})$. A significant increase in $\triangle$ Tau-positive cells (red) was observed after 6 hours $\left({ }^{\star} P=0.012\right)$ and 24 hours $\left({ }^{\star *} P<0.0001\right)$ of $A \beta$ treatment (D). The great majority of $\Delta$ Tau-positive cells (91.5\%) contained apoptotic nuclei as revealed by DAPI (A-C). In addition, $\Delta$ Tau immunolabeling also revealed striate tangle-like morphology in association with apoptotic nuclei (B and C). Scale bar: $9 \mu \mathrm{m}$ (A), $5 \mu \mathrm{m}$ (B), $7.5 \mu \mathrm{m}$ (C).

Although isolated PHFs contain truncated tau species (e.g., truncation at Glu391) that may play a role in NFT formation $(47,48)$, the mechanism(s) that gives rise to tau truncation is currently unknown. The presence of $\Delta$ Tau throughout the progression of tangle pathology suggests that truncated tau species may arise from further proteolysis of $\Delta \mathrm{Tau}$. However, the presence of both full-length tau (T46) and $\Delta$ Tau in NFTs implicates a role for both full-length and caspase-cleaved tau in tangle formation. Although $\Delta$ Tau colocalized with several NFT markers in the AD brain, we have not yet demonstrated the presence of $\Delta$ Tau within PHFs at the ultrastructural level. Future experiments aim to address this caveat.

Hyperphosphorylation of tau is the prevailing hypothesis in the development of tangle pathology, since hyperphosphorylation can promote PHF self-assembly (49). Transgenic animal models (mice, Caenorbabditis elegans, Drosophila) strengthen this hypothesis (50-54). Although no previous mechanism has been described linking caspase-cleavage and the hyperphosphorylation of tau, the appearance of hyperphosphorylated tau following the activation of apoptosis suggests that the two events may be closely connected (55). Our data show that $\Delta$ Tau can be hyperphosphorylated after

\section{Figure 10}

$\Delta$ Tau colocalizes with pathological features of a triple-transgenic model of $A D$. Fluorescent double labeling of $3 x T g-A D$ mice demonstrates that intraneuronal $A \beta$ (green) colocalizes with $\Delta T a u$ (red) within CA1 pyramidal neurons of 12-month-old (A) and 18-month-old (B) 3xTg-AD mice and within 18-month-old neocortex (C). $\Delta$ Tau immunoreactivity (red) also colocalizes with an early tangle marker, MC1 (green), within CA1 pyramidal neurons at both 12-month (D) and 18-month time points ( $E$ and F). Scale bar: $30 \mu \mathrm{m}$ (A, D, and E), $20 \mu \mathrm{m}$ (B), $4 \mu \mathrm{m}$ (C and F). caspase-cleavage, therefore suggesting that production of $\Delta \mathrm{Tau}$ does not preclude subsequent hyperphosphorylation.

Recent evidence suggests that $\mathrm{A} \beta$ accumulation precedes NFT formation in the AD brain and can potentiate NFTs in transgenic mice $(45,51,52,56-58)$. Furthermore, A $\beta$-induced neurodegeneration requires tau expression and may involve the intracellular assembly of pathological tau filaments $(59,60)$. The colocalization of $\Delta$ Tau with both intraneuronal and extracellular $\mathrm{A} \beta$ deposits suggests that $\mathrm{A} \beta$ accumulation may initiate caspasecleavage of tau. The granular appearance of intracellular $A \beta$ is also observed in Down syndrome patients with AD (61), indicating that intraneuronal $\mathrm{A} \beta$ may be a common feature of AD subtypes. It has been proposed that intraneuronal $A \beta$ accumulation may precede extracellular-plaque pathology in both $\mathrm{AD}$ and transgenic mouse models $(45,57,61-63)$, further suggesting that caspasecleavage of tau may occur early in AD pathology. However, the notion that intracellular $\mathrm{A} \beta$ precedes extracellular-plaque deposition remains controversial.

Functional decline in $\mathrm{AD}$ correlates with increasing tangle pathology $(56,64-66)$. The production of $\Delta$ Tau correlated with the clinical progression of AD. In addition to possibly initiating NFT pathology, caspase-cleavage of tau may contribute to neuronal dysfunction. 3xTg-AD mice develop synaptic dysfunction that coincides with intracellular $\mathrm{A} \beta$ accumulation (45). Because intracellular $\mathrm{A} \beta$ colocalized with caspase-cleaved tau in the brains of both subjects with $\mathrm{AD}$ and $3 \mathrm{xTg}-\mathrm{AD}$ mice, $\Delta \mathrm{Tau}$ may contribute to further synaptic dysfunction and loss, in vivo. In a C. elegans model of tau neurodegenerative disease that displays defects in cholinergic transmission, Kraemer et al. detected a truncated tau species (50). It is interesting to speculate that this truncation may be due to caspasecleavage and may contribute to the observed synaptic dysfunction.

$\mathrm{AD}$ is also characterized by the activation of apoptotic pathways. Although the hypothesis that apoptosis plays a role in $\mathrm{AD}$ neurodegeneration remains controversial, recent evidence suggests that caspase activation can occur independent of cell death and may be neuroprotective (67-69). In addition, caspase activation is implicated in long-term potentiation and neurite extension (70). However, caspase-cleaved tau leads to neurite retraction in vitro (71) and thus may contribute to synaptic deficits and cellular demise. Although the mechanisms that trigger caspases in the AD brain are currently unknown, $\mathrm{A} \beta$, which accumulates in the $\mathrm{AD}$ brain, is one of many known activators of apoptosis $(42,72)$. Because caspase activation is associated with NFT pathology $(20-23,25,29)$, our data support
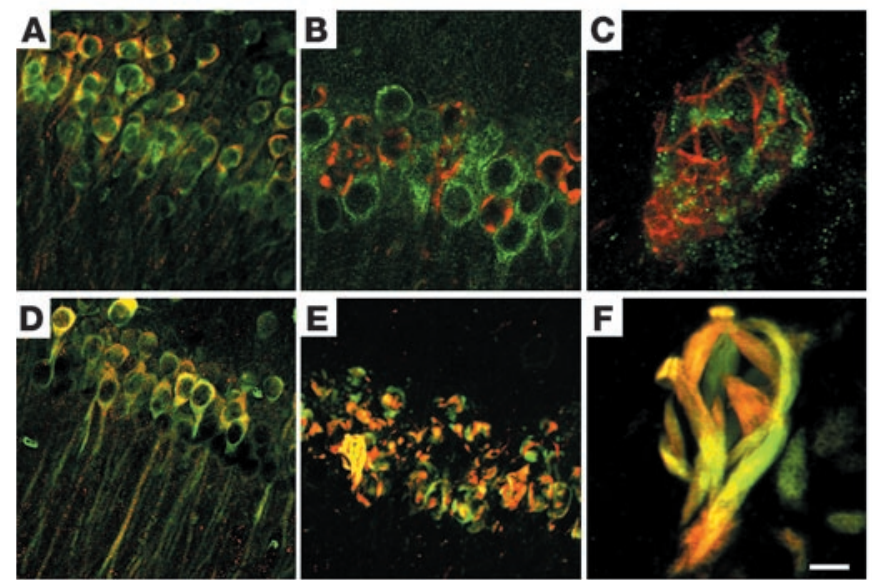


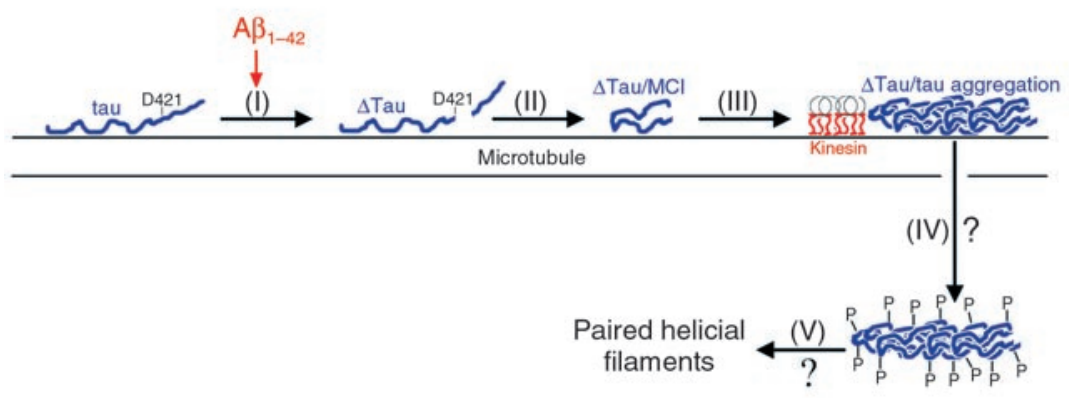

Figure 11

Proposed model for the evolution of NFTs. Apoptotic stimuli such as $A \beta_{1-42}$ exposure result in caspase-cleavage of tau after D421 (I). Caspase-cleaved tau rapidly adopts the MC1 conformational epitope (II), which leads to increased filament formation and tau aggregation (III). To compensate for tau aggregation, tau may subsequently be hyperphosphorylated and disassociate from the microtubule (IV). As a result, caspasecleavage of tau may lead to PHF formation (V).

the idea that $A \beta$-induced caspase activation may lead to pathological tau $(34,73)$. Thus, in $\mathrm{AD}, \mathrm{A} \beta$ and tau pathologies may be closely linked through a common pathway, caspase-mediated proteolysis. Furthermore, we observed that $\Delta$ Tau immunoreactivity defined striking tangle-like structures in degenerating neurons, which is suggestive of $A \beta$ contributing to tangle pathology $(51,52,58$, 74). Interestingly, in tau transgenic Drosophila, inhibitors of apoptosis suppressed tau-induced neurodegeneration (53). Although tau is also hyperphosphorylated in this model, the inhibition of neurodegeneration underlies the importance of caspase activation and possibly tau caspase-cleavage in this animal model.

Finally, our results are in agreement with a recent study demonstrating that $A \beta$ leads to caspase-cleavage of tau (34). The authors' findings and ours support a model in which any stimuli capable of activating caspases (e.g., oxidative damage, neurotrophic factor deprivation, low energy metabolism, mitochondrial dysfunction) can initiate NFT pathology. Our preliminary data indicate that 4-hydroxynonenal, an inducer of oxidative stress and activator of caspases, leads to the production of $\Delta T$ Tau in rat primary neurons (data not shown). Thus, we have proposed a model describing the role of caspase-cleavage of tau in the development of tangle pathology (Figure 11). Following any stimulus that results in caspase activation (e.g., $A \beta_{1-42}$ ), tau is cleaved at D421 (step 1). Upon caspase-cleavage, tau adopts an MC1-immunoreactive conformation (step 2), which leads to increased filament formation that may occur on microtubules (where tau binds in vivo) (step 3) (75). Although we do not specifically examine whether caspase-cleaved tau results in deficits in kinesin-mediated transport, we speculate that $\Delta$ Tau may block kinesin-mediated anterograde transport akin to the overexpression of tau $(2,3)$. As a protective mechanism in order to compensate (relieve the blockage), phosphorylation of tau occurs to dissociate it from microtubules (step 4) (16-18). Finally, dissociation of phosphorylated tau species leads to destabilization of the microtubule network and PHF formation (step 5). Although destabilization of microtubules may initially be neuroprotective (59), it will eventually lead to the demise of the cell. Our mechanism does not exclude other reported mechanisms leading to tau hyperphosphorylation but suggests that caspase-cleavage of tau contributes to tau hyperphosphorylation in AD.

Thus, in neurodegenerative diseases where tau pathology occurs in the absence of A $\beta$ (e.g., Pick disease and supranuclear palsy), other stimuli known to activate caspases may be involved in the initiation of NFT pathology. Therefore, caspase-cleavage of tau may play an important role not only in $\mathrm{AD}$, but also in other tauopathies, and may serve as a key target for the development of novel therapeutics.

\section{Methods}

In vitro caspase-cleavage of tau. To examine the specificity of caspase-cleavage of tau, $25 \mu \mathrm{g}$ of recombinant human tau40 (Calbiochem, San Diego, California, USA) was incubated with active human recombinant caspase- $1,-3,-4,-5,-7,-8$, or -10 (Calbiochem; and AG Scientific Inc., San Diego, California, USA). The caspase-specific activity was adjusted so that all caspases would cleave $1 \mathrm{nmol}$ of substrate per hour at $37^{\circ} \mathrm{C}$. Caspase digestion was performed ( 4 hours, $37^{\circ} \mathrm{C}$ ), and cleavage reactions were terminated by addition of $10 \%$ formic acid $(1 \mu \mathrm{l})$. Full-length or caspase-treated recombinant tau (30 ng) was separated by $10 \%$ SDS-PAGE and either stained with Coomassie blue or analyzed by Western blot, as described below.

Electrospray mass spectrometry. Samples were analyzed using an HPLC-Micromass Q-TOF 2 (Waters, Milford, Massachusetts, USA) using a hand-packed C8 silica column. The flow rate was split from $100 \mu \mathrm{l}$ to $400 \mathrm{nl} / \mathrm{min}$ over a 60 -minute gradient (3-70\% acetonitrile). Deconvolution of the spectra was performed to identify protein masses (MaxEnt 1 software; Micromass, Manchester, United Kingdom).

Generation of $\alpha-\Delta$ Tau polyclonal antibody. The peptide SSTGSIDMVD corresponding to the carboxy-terminus of tau following caspase-cleavage was conjugated to keyhole limpet hemocyanin and injected into rabbits. Research Genetics Inc. (Huntsville, Alabama, USA) performed the synthesis of peptides, injections of immunogens, and collection of antisera. The peptide CSTGSIDMVD was covalently linked to a gel column (SulfoLink kit; Pierce Biotechnology Inc., Rockford, Illinois, USA) and used to affinitypurify antisera from individual rabbits.

Transgenic mice. All rodent experiments were performed in accordance with animal protocols approved by the Institutional Animal Care and Use Committee at either the University of California, Irvine (UCI), or Duke University (TBI experiments). The tripletransgenic mice (3xTg-AD) have been described previously (45). Briefly, these mice harbor a knock-in mutation of presenilin 1 $\left(P S 1_{M 146 V}\right)$, the Swedish double mutation of amyloid precursor protein $\left(A P P_{K M 670 / 671 N L}\right)$, and a frontotemporal dementia mutation in tau $\left(\tan _{P 301 L}\right)$ on a $129 / \mathrm{C} 57 \mathrm{BL} / 6$ background. Homozygous mice were sacrificed by $\mathrm{CO}_{2}$ asphyxiation at 6-month, 12-month, and 18 -month time points, and brains were rapidly removed and immersion-fixed in $4 \%$ paraformaldehyde for 48 hours. Coronal sections were cut on a Vibratome (Technical Products International, St. Louis, Missouri, USA) $(50 \mu \mathrm{m})$ and stored in PBS, $0.02 \%$ $\mathrm{NaN}_{3}\left(4^{\circ} \mathrm{C}\right)$, until use. Tau-/- mice (129/C57BL/6 background) have been previously described (76). Closed-head injury was performed in male tau knockout and wild-type mice (age 12-14 weeks) with a pneumatic impactor as previously described (77). Forty-eight hours after injury, mice were deeply anesthetized and transcardially perfused (4\% paraformaldehyde). Following postfixation, brains were cut and stored as detailed above. All mice used in this study were maintained and housed in accordance 
with NIH and Association for Assessment and Accreditation of Laboratory Animal Care guidelines.

Human subjects. Postmortem human brain tissues were obtained from the Institute for Brain Aging and Dementia Tissue Repository at UCI. A total of 12 neuropathologically confirmed AD cases, three cases with MCI, and 11 nondemented control cases, matched for both mean age and postmortem interval, were examined. Case demographics are presented in Supplemental Table 1. Brain sections fixed in 4\% paraformaldehyde and containing the midregion of the hippocampus and entorhinal cortex were cut as described above. For Western analysis, superior temporal gyri from control, MCI, and AD cases underwent sequential fractionation, as described below. We also included a transitional $\mathrm{AD}$ case ( $\left.{ }^{*} \mathrm{MCI}\right)$. This patient fell outside the normal range of MCI but did not meet the criteria for $\mathrm{AD}$ dementia.

Clinical diagnoses. Control cases exhibited no memory impairment and were normal on tests of functional ability. MCI was diagnosed by neuropsychological and neurological examination (at the Institute for Brain Aging and Dementia, UCI) as previously described (78). MCI individuals were not demented but had memory decline and scored at least 1.5 SD below the mean of their age-matched peers on objective tests of episodic memory (e.g., Consortium to Establish a Registry for Alzheimer Disease [CERAD]; Word list test, Logical memory test). In addition, these individuals performed within normal limits on all other tests of cognitive functioning and had no significant deficits in measures of functional ability (e.g., Dementia Rating Severity Scale and Functional Activity Questionnaire).QuestionnaireAD cases were severely impaired in memory tasks and measures of functional ability and daily living.

Immunohistochemistry and confocal microscopy. Light-level immunohistochemistry was performed using an avidin-biotin immunoperoxidase technique (ABC kit; Vector Laboratories Inc., Burlingame, California, USA) and was visualized with diaminobenzidine as previously described (22). Fluorescent immunolabeling followed either a standard two-way technique (primary antibody followed by fluorescent secondary antibody) or a three-way protocol (primary antibody, biotinylated secondary antibody, and then streptavidin-conjugated fluorophore). Free-floating sections were rinsed in PBS ( $\mathrm{pH} 7.4$ ) and then blocked (0.25\% Triton X-100, $5 \%$ normal goat serum in PBS) for 1 hour. Sections were incubated in primary antibody overnight $\left(4^{\circ} \mathrm{C}\right)$, rinsed in PBS, and incubated (1 hour) in either fluorescently labeled anti-rabbit or anti-mouse secondary antibodies (Alexa 488 or 568, 1:200; Molecular Probes Inc., Eugene, Oregon, USA) or biotinylated secondary antibodies (1:150; Vector Laboratories Inc.) followed by streptavidin-Alexa 488 (1:200). When two monoclonal or two polyclonal antibodies were used in combination (e.g., MC1 and PHF-1), one antibody was detected using a standard fluorescent secondary antibody. The second epitope was detected by direct conjugation of a fluorophore to the primary antibody (Zenon kit; Molecular Probes Inc.). Antibodies were diluted as follows: $\Delta$ Tau, 1:1,000; T46, 1:1,000 (Zymed Laboratories Inc., South San Francisco, California, USA); active caspase-3, 1:100 (Promega Corp., Madison, Wisconsin, USA); caspase-cleaved fodrin, 1:100 (Cell Signaling Technology Inc., Beverly, Massachusetts, USA); MC1 and PHF-1, 1:500 (gifts of P. Davies, Yeshiva University, Bronx, New York, USA); A $\beta_{1-42}$, 1:100 (Biosource International Inc., Camarillo, California, USA); $\mathrm{A} \beta_{17-24}$ (clone 4G8), 1:100 (Chemicon International Inc., Temecula, California, USA); MAP-2, 1:2,000 (Sigma-Aldrich, St. Louis, Missouri, USA). DAPI (1:400; Molecular Probes Inc.) was used to assess apoptotic nuclei in vitro. Omission of primary antibody or use of preimmune IgG eliminated all labeling (data not shown). In addition, preadsorption of $\alpha-\Delta$ Tau antibody with an excess of immunizing peptide resulted in no immunoreactivity (Figure $3 \mathrm{~A}$, inset). Confocal images were captured on FluoView (Olympus America Inc., Melville, New York, USA) or MRC 1024 (Bio-Rad, Hercules, California, USA) confocal systems. In some cases images were pseudocolored to maintain consistent use of color for a given antigen throughout all figures. To prevent signal bleed-through, all fluorophores were excited and scanned separately using appropriate laser lines and filter sets.

Quantification of $\triangle$ Tau and correlation with MMSE score. Using the human postmortem tissues described above, the relationship between $\Delta$ Tau-immunoreactive cell number and cognitive function was examined. Sections from $\mathrm{AD}$ and control cases $(n=8)$ were immunolabeled for $\Delta$ Tau using a standard avidin-biotin immunoperoxidase technique. The number of $\Delta$ Tau-positive cells within control and AD sections was counted from $\times 20$ photographs of two randomly chosen regions of CA1. Cell counts were averaged and statistically compared by Student's $t$ test. The relationship between $\Delta$ Tau-positive cell number and MMSE score was then determined by Spearman's correlation test. Only AD cases were included in the analysis, since control cases can act as ordinate values and artificially increase correlation strength and significance. All statistical analyses were performed using StatView 5.1 (SAS Institute Inc., Cary, North Carolina, USA).

Analysis of $\Delta$ Tau solubility. Superior temporal gyri of control, MCI, and $\mathrm{AD}$ cases were processed to isolate RAB and RIPA fractions using previously described protocols $(50,79)$. Briefly, frozen tissue was homogenized in $2 \mathrm{ml} / \mathrm{g}$ of RAB-HS (0.1 M 2-(N-morpholino) [MES], $1 \mathrm{mM}$ EGTA, $0.5 \mathrm{mM} \mathrm{MgSO}_{4}, 0.75 \mathrm{M} \mathrm{NaCl}, 0.02 \mathrm{M}$ $\mathrm{NaF}, 1 \mathrm{mM}$ PMSF, and $1 \%$ protease inhibitor cocktail [P8340; Sigma-Aldrich]) and centrifuged at 40,000 $\mathrm{g}$ for 40 minutes, and the supernatant (RAB fraction) was collected. The RAB-HS pellets were rehomogenized in RAB-HS containing $1 \mathrm{M}$ sucrose and centrifuged at 40,000 $\mathrm{g}$ for 20 minutes. The pellets were subsequently resuspended in RIPA (50 mM Tris, $150 \mathrm{mM} \mathrm{NaCl}, 0.1 \%$ SDS, $0.5 \%$ Na deoxycholate, 1\% NP-40, 5 mM EDTA, 1 mM PMSF, $1 \mathrm{mM}$ $\mathrm{NaF}, 1 \mathrm{mM} \mathrm{Na} \mathrm{VO}_{4}$, and $1 \mu \mathrm{g} / \mathrm{ml}$ aprotinin, leupeptin, and pepstatin) at $1 \mathrm{ml} / \mathrm{g}$ and centrifuged at 40,000 $\mathrm{g}$ for 20 minutes, and the resulting RIPA-soluble supernatant was collected. BCA protein assay (Pierce Biotechnology Inc.) was performed to determine protein concentration, and equal protein amounts of RAB and RIPA fractions were then analyzed by Western blot.

Western blot analysis. Recombinant tau proteins and brain lysates were processed for Western blot. Briefly, proteins were separated by $10 \%$ SDS-PAGE and transferred to nitrocellulose $(0.2 \mu \mathrm{m}$; Schleicher $\&$ Schuell BioScience Inc., Keene, New Hampshire, USA). Membranes were incubated in either $\alpha-\Delta \operatorname{Tau}(1: 1,000)$ or T46 $(1: 1,000)$. Primary antibody was visualized using either goat anti-rabbit or anti-mouse HRP-linked secondary antibody (1:1,000; Calbiochem), followed by ECL detection (Pierce Biotechnology Inc.).

Electron microscopy. Tissue from AD cases was embedded in Lowicryl (Ted Pella Inc., Redding, California, USA), and ultrathin sections were immunolabeled using a post-embedding procedure (80) with $\Delta$ Tau primary antibody (1:300), followed goat anti-rabbit IgG that was previously conjugated to $10 \mathrm{~nm}$ gold particles (1:20; Ted Pella Inc.). Following incubation in secondary antibody, the sections were counterstained with uranyl acetate and lead citrate and examined with a Philips CM 10 electron microscope (Phillips Electronic Instruments, Mahwah, New Jersey, USA). 
Laser light scattering. Laser light scattering (LLS) was performed as previously described with slight modifications $(38,40,47)$. Briefly, tau $(4 \mu \mathrm{M})$ was resuspended in $10 \mathrm{mM}$ HEPES ( $\mathrm{pH} 7.4), 16$ $\mu \mathrm{M}$ heparin, $5 \mathrm{mM}$ DTT, and $0.1 \% \mathrm{NaN}_{3}$, and the amount of scattered light was assessed. The degree of light scattering was monitored (room temperature, 400 minutes) in a Fluorometer Micro Cell (3 mm; Starna Cells Inc.) illuminated by a He-Ne laser (632 $\mathrm{nm})$. Light scattering was recorded with a charged coupled device (CCD) digital video camera (PL-A641; PixeLINK, Ottawa, Ontario, Canada) attached to a microscope objective $(\times 4)$ positioned at $90^{\circ}$ to the direction of the incident laser beam. Resultant scatter signal intensities were subtracted from signal adjacent to the nonilluminated area (background subtraction). LLS recording software was written by W.E. van der Veer (UCI Chemistry Core Facility) using LabView (National Instruments, Austin, Texas, USA).

MC1 immunoprecipitation. Full-length and caspase-3-cleaved recombinant tau was immunoprecipitated overnight $\left(4^{\circ} \mathrm{C}\right)$ with $\mathrm{mAb}$ MC1 coupled to protein G (Pierce Biotechnology Inc.). Immunoprecipitated proteins were eluted with sample buffer and analyzed by Western blot using a rabbit polyclonal anti-tau primary antibody (1:1,000; gift of L.I. Binder). Membranes were then incubated with HRP-linked anti-rabbit secondary antibody (1:1,000; Calbiochem) and detected with chromogen 4CN (PerkinElmer Inc., Massachusetts, USA).

In vitro phosphorylation assay. Full-length or caspase-3-cleaved recombinant tau was purified using TALON metal affinity resin (BD Biosciences - Clontech, Palo Alto, California, USA) to remove active caspase-3 prior to in vitro phosphorylation assays. All reactions were carried out at $37^{\circ} \mathrm{C}$ in $40 \mathrm{mM}$ HEPES ( $\mathrm{pH} 7.2$ ), $3 \mathrm{mM} \mathrm{MgCl}_{2}, 5 \mathrm{mM}$ EGTA, and $2 \mathrm{mM}$ ATP. Fresh ATP was added immediately before GSK-3 $\beta$ (Calbiochem). Aliquots were removed at various time points, and reactions were stopped by the addition of sample buffer, boiled at $100^{\circ} \mathrm{C}$ (10 minutes), and analyzed by Western blot.

$A \beta$ treatment of rat cortical neurons. Primary cortical neurons (E18) were plated on poly-L-lysine-coated coverslips $\left(1.5 \times 10^{5}\right.$ cells per coverslip). After 7 days in culture, cells were treated with $25 \mu \mathrm{M}$ fibrillar $A \beta_{1-42}$ (Biosource International Inc.) for $1,4,6$, or 24 hours. Fibrillar $A \beta_{1-42}$ was prepared by freeze-thawing (three times) followed by incubation at room temperature overnight. Following treatment, cells were fixed in $4 \%$ paraformaldehyde and processed for immunocytochemistry as described above. The number of $\triangle \mathrm{Tau}, \mathrm{MAP}-2$, and $\Delta$ Tau cells with DNA fragmentation (DAPI) within a defined counting frame $\left(500 \mu \mathrm{m}^{2}\right)$ were quantified with a $\times 20$ objective under epifluorescence illumination by a blinded observer. Four randomly chosen regions were counted from two coverslips per treatment. Data were analyzed using ANOVA for a split-plot design followed by Fisher's protected least significant difference post hoc analysis. All statistical analyses were performed using StatView 5.1 (SAS Institute Inc.).

\section{Acknowledgments}

This work was supported in part by NIH/National Institute on Aging grants AG000096 (to R.A. Rissman, W.W. Poon, and M. Blurton-Jones), AG023433 (to R.A. Rissman), AG000538 and P50 AG016573 (to C.W. Cotman), AG17968 (to F.M. LaFerla), AG19386 (to T.T. Rohn), and AG19780 (to M.P. Vitek). We thank P.A. Adlard, E. Head, and D.H. Cribbs (Institute for Brain Aging and Dementia, UCI) for critical reading of the manuscript. We are grateful to J.P. Kesslak and W.E. van der Veer (Department of Chemistry, UCI) for statistical advice and LLS assistance, respectively. We would also like to thank Matthew D.E. Loper for his invaluable contributions.

Received for publication November 25, 2003, and accepted in revised form May 7, 2004.

Address correspondence to: Carl W. Cotman, Institute for Brain Aging and Dementia, University of California, Irvine, 1113 Gillespie, Neuroscience Research Facility, Irvine, California 92697, USA. Phone: (949) 824-5847; Fax: (949) 824-2071; E-mail: cwcotman@uci.edu.

Robert A. Rissman, Wayne W. Poon, and Mathew Blurton-Jones contributed equally to this work.
1. Caceres, A., and Kosik, K.S. 1990. Inhibition of neurite polarity by tau antisense oligonucleotides in primary cerebellar neurons. Nature. 343:461-463.

2. Ebneth, A., et al. 1998. Overexpression of tau protein inhibits kinesin-dependent trafficking of vesicles, mitochondria, and endoplasmic reticulum: implications for Alzheimer's disease. J. Cell Biol. 143:777-794.

3. Stamer, K., Vogel, R., Thies, E., Mandelkow, E., and Mandelkow, E.M. 2002. Tau blocks traffic of organelles, neurofilaments, and APP vesicles in neurons and enhances oxidative stress. J. Cell Biol. 156:1051-1063.

4. Buee, L., Bussiere, T., Buee-Scherrer, V., Delacourte, A., and Hof, P.R. 2000. Tau protein isoforms, phosphorylation and role in neurodegenerative disorders. Brain Res. Brain Res. Rev. 33:95-130.

5. Lee, V.M., Goedert, M., and Trojanowski, J.Q. 2001. Neurodegenerative tauopathies. Annu. Rev. Neurosci. 24:1121-1159.

6. Grundke-Iqbal, I., et al. 1986. Microtubule-associated protein tau. A component of Alzheimer paired helical filaments. J. Biol. Chem. 261:6084-6089.

7. Wischik, C.M., et al. 1988. Structural characterization of the core of the paired helical filament of Alzheimer disease. Proc. Natl. Acad. Sci. U. S. A. 85:4884-4888.

8. Braak, H., and Braak, E. 1991. Neuropathological stageing of Alzheimer-related changes. Acta
Neuropathol. 82:239-259.

9. Jicha, G.A., Bowser, R., Kazam, I.G., and Davies, P. 1997. Alz-50 and MC-1, a new monoclonal antibody raised to paired helical filaments, recognize conformational epitopes on recombinant tau. J. Neurosci. Res. 48:128-132.

10. Jicha, G.A., Berenfeld, B., and Davies, P. 1999. Sequence requirements for formation of conformational variants of tau similar to those found in Alzheimer's disease. J. Neurosci. Res. 55:713-723.

11. Uboga, N.V., and Price, J.L. 2000. Formation of diffuse and fibrillar tangles in aging and early Alzheimer's disease. Neurobiol. Aging. 21:1-10.

12. Weaver, C.L., Espinoza, M., Kress, Y., and Davies, P. 2000. Conformational change as one of the earliest alterations of tau in Alzheimer's disease. Neurobiol. Aging. 21:719-727.

13. Goedert, M., Jakes, R., and Vanmechelen, E. 1995. Monoclonal antibody AT8 recognises tau protein phosphorylated at both serine 202 and threonine 205. Neurosci. Lett. 189:167-169.

14. Goedert, M., et al. 1993. The abnormal phosphorylation of tau protein at Ser-202 in Alzheimer disease recapitulates phosphorylation during development. Proc. Natl. Acad. Sci. U. S. A. 90:5066-5070.

15. Greenberg, S.G., Davies, P., Schein, J.D., and Binder, L.I. 1992. Hydrofluoric acid-treated tau PHF proteins display the same biochemical properties as normal tau. J. Biol. Chem. 267:564-569.

16. Drechsel, D.N., Hyman, A.A., Cobb, M.H., and Kirschner, M.W. 1992. Modulation of the dynamic instability of tubulin assembly by the microtubuleassociated protein tau. Mol. Biol. Cell. 3:1141-1154.

17. Biernat, J., Gustke, N., Drewes, G., Mandelkow, E.M., and Mandelkow, E. 1993. Phosphorylation of Ser262 strongly reduces binding of tau to microtubules: distinction between PHF-like immunoreactivity and microtubule binding. Neuron. 11:153-163.

18. Bramblett, G.T., et al. 1993. Abnormal tau phosphorylation at Ser396 in Alzheimer's disease recapitulates development and contributes to reduced microtubule binding. Neuron. 10:1089-1099.

19. Kerr, J.F., Wyllie, A.H., and Currie, A.R. 1972. Apoptosis: a basic biological phenomenon with wide-ranging implications in tissue kinetics. $\mathrm{Br}$. $\mathrm{J}$. Cancer. 26:239-257.

20. Gervais, F.G., et al. 1999. Involvement of caspases in proteolytic cleavage of Alzheimer's amyloidbeta precursor protein and amyloidogenic A beta peptide formation. Cell. 97:395-406.

21. Stadelmann, C., et al. 1999. Activation of caspase3 in single neurons and autophagic granules of granulovacuolar degeneration in Alzheimer's disease. Evidence for apoptotic cell death. Am. J. Pathol. 155:1459-1466.

22. Rohn, T.T., Head, E., Nesse, W.H., Cotman, C.W., 
and Cribbs, D.H. 2001. Activation of caspase-8 in the Alzheimer's disease brain. Neurobiol. Dis. 8:1006-1016

23. Rohn, T.T., et al. 2001. Correlation between caspase activation and neurofibrillary tangle formation in Alzheimer's disease. Am. J. Pathol. 158:189-198.

24. Su, J.H., Zhao, M., Anderson, A.J., Srinivasan, A., and Cotman, C.W. 2001. Activated caspase-3 expression in Alzheimer's and aged control brain correlation with Alzheimer pathology. Brain Res. 898:350-357.

25. Rohn, T.T., et al. 2002. Caspase- 9 activation and caspase cleavage of tau in the Alzheimer's disease brain. Neurobiol. Dis. 11:341-354.

26. Gastard, M.C., Troncoso, J.C., and Koliatsos, V.E. 2003. Caspase activation in the limbic cortex of subjects with early Alzheimer's disease. Ann. Neurol. 54:393-398.

27. Martin, S.J., et al. 1995. Proteolysis of fodrin (nonerythroid spectrin) during apoptosis. J. Biol. Chem. 270:6425-6428.

28. Canu, N., et al. 1998. Tau cleavage and dephosphorylation in cerebellar granule neurons undergoing apoptosis. J. Neurosci. 18:7061-7074.

29. Yang, F., et al. 1998. Antibody to caspase-cleaved actin detects apoptosis in differentiated neuroblastoma and plaque-associated neurons and microglia in Alzheimer's disease. Am. J. Pathol. 152:379-389.

30. Fasulo, L., et al. 2000. The neuronal microtubuleassociated protein tau is a substrate for caspase- 3 and an effector of apoptosis. J. Neurochem. 75:624-633.

31. Chung, C.W., et al. 2001. Proapoptotic effects of tau cleavage product generated by caspase- 3 . Neurobiol. Dis. 8:162-172.

32. Lee, J.C., et al. 2002. DEDD regulates degradation of intermediate filaments during apoptosis. J. Cell Biol. 158:1051-1066.

33. Utsumi, T., Sakurai, N., Nakano, K., and Ishisaka, R. 2003. C-terminal $15 \mathrm{kDa}$ fragment of cytoskeletal actin is posttranslationally $\mathrm{N}$-myristoylated upon caspase-mediated cleavage and targeted to mitochondria. FEBS Lett. 539:37-44.

34. Gamblin, T.C., et al. 2003. Caspase cleavage of tau: linking amyloid and neurofibrillary tangles in Alzheimer's disease. Proc. Natl. Acad. Sci. U. S. A 100:10032-10037.

35. Carmel, G., Mager, E.M., Binder, L.I., and Kuret, J. 1996. The structural basis of monoclonal antibody Alz50's selectivity for Alzheimer's disease pathology. J. Biol. Chem. 271:32789-32795.

36. Kosik, K.S., et al. 1988. Epitopes that span the tau molecule are shared with paired helical filaments. Neuron. 1:817-825.

37. Knoblach, S.M., et al. 2002. Multiple caspases are activated after traumatic brain injury: evidence fo involvement in functional outcome. J. Neurotrauma. 19:1155-1170.

38. Gamblin, T.C., King, M.E., Kuret, J., Berry, R.W. and Binder, L.I. 2000. Oxidative regulation of fatty acid-induced tau polymerization. Biochemistry. 39:14203-14210.

39. Barghorn, S., and Mandelkow, E. 2002. Toward a unified scheme for the aggregation of tau into Alzheimer paired helical filaments. Biochemistry. 41:14885-14896.

40. Friedhoff, P., von Bergen, M., Mandelkow, E.M., Davies, P., and Mandelkow, E. 1998. A nucleated assembly mechanism of Alzheimer paired helical filaments. Proc. Natl. Acad. Sci. U. S. A. 95:15712-15717.

41. Lovestone, S., et al. 1994. Alzheimer's disease-like phosphorylation of the microtubule-associated protein tau by glycogen synthase kinase- 3 in transfected mammalian cells. Curr. Biol. 4:1077-1086.
42. Loo, D.T., et al. 1993. Apoptosis is induced by betaamyloid in cultured central nervous system neurons. Proc. Natl. Acad. Sci. U. S. A. 90:7951-7955.

43. Ivins, K.J., Ivins, J.K., Sharp, J.P., and Cotman, C.W. 1999. Multiple pathways of apoptosis in PC12 cells. CrmA inhibits apoptosis induced by beta-amyloid. J. Biol. Chem. 274:2107-2112.

44. Ivins, K.J., Thornton, P.L., Rohn, T.T., and Cotman, C.W. 1999. Neuronal apoptosis induced by beta-amyloid is mediated by caspase- 8 . Neurobiol. Dis. 6:440-449.

45. Oddo, S., et al. 2003. Triple-transgenic model of Alzheimer's disease with plaques and tangles: intracellular Abeta and synaptic dysfunction. New ron. 39:409-421.

46. Berry, R.W., et al. 2003. Inhibition of tau polymerization by its carboxy-terminal caspase cleavage fragment. Biochemistry. 42:8325-8331.

47. Abraha, A., et al. 2000. C-terminal inhibition of tau assembly in vitro and in Alzheimer's disease. J. Cell Sci. 113:3737-3745

48. Novak, M., Kabat, J., and Wischik, C.M. 1993. Molecular characterization of the minimal protease resistant tau unit of the Alzheimer's disease paired helical filament. EMBOJ. 12:365-370.

49. Alonso, A., Zaidi, T., Novak, M., Grundke-Iqbal, I., and Iqbal, K. 2001. Hyperphosphorylation induces self-assembly of tau into tangles of paired helical filaments/straight filaments. Proc. Natl. Acad. Sci. U. S. A. 98:6923-6928.

50. Kraemer, B.C., et al. 2003. Neurodegeneration and defective neurotransmission in a Caenorhabditis elegans model of tauopathy. Proc. Natl. Acad. Sci. U. S. A. 100:9980-9985.

51. Gotz, J., Chen, F., van Dorpe, J., and Nitsch, R.M. 2001. Formation of neurofibrillary tangles in P301 tau transgenic mice induced by Abeta 42 fibrils. Science. 293:1491-1495.

52. Lewis, J., et al. 2001. Enhanced neurofibrillary degeneration in transgenic mice expressing mutant tau and APP. Science. 293:1487-1491.

53. Jackson, G.R., et al. 2002. Human wild-type tau interacts with wingless pathway components and produces neurofibrillary pathology in Drosophila. Neuron. 34:509-519.

54. Andorfer, C., et al. 2003. Hyperphosphorylation and aggregation of tau in mice expressing normal human tau isoforms. J. Neurochem. 86:582-590.

55. Mookherjee, P., and Johnson, G.V. 2001. Tau phosphorylation during apoptosis of human $\mathrm{SH}$ SY5Y neuroblastoma cells. Brain Res. 921:31-43.

56. Mukaetova-Ladinska, E.B., et al. 2000. Staging of cytoskeletal and beta-amyloid changes in human isocortex reveals biphasic synaptic protein response during progression of Alzheimer's disease. Am. J. Pathol. 157:623-636.

57. Gouras, G.K., et al. 2000. Intraneuronal Abeta42 accumulation in human brain. Am. J. Pathol. 156:15-20.

58. Oddo, S., Caccamo, A., Kitazawa, M., Tseng, B.P., and LaFerla, F.M. 2003. Amyloid deposition precedes tangle formation in a triple transgenic model of Alzheimer's disease. Neurobiol. Aging. 24:1063-1070.

59. Rapoport, M., Dawson, H.N., Binder, L.I., Vitek, M.P., and Ferreira, A. 2002. Tau is essential to betaamyloid-induced neurotoxicity. Proc. Natl. Acad. Sci. U. S. A. 99:6364-6369.

60. Sponne, I., et al. 2003. Apoptotic neuronal cell death induced by the non-fibrillar amyloid-beta peptide proceeds through an early reactive oxygen species-dependent cytoskeleton perturbation. J. Biol. Chem. 278:3437-3445.

61. Busciglio, J., et al. 2002. Altered metabolism of the amyloid beta precursor protein is associated with mitochondrial dysfunction in Down's syndrome. Neuron. 33:677-688

62. Takahashi, R.H., et al. 2002. Intraneuronal Alzheimer abeta42 accumulates in multivesicular bodies and is associated with synaptic pathology. Am. J. Pathol. 161:1869-1879.

63. Wirths, O., et al. 2001. Intraneuronal Abeta accumulation precedes plaque formation in betaamyloid precursor protein and presenilin-1 double-transgenic mice. Neurosci. Lett. 306:116-120

64. McKee, A.C., Kosik, K.S., and Kowall, N.W. 1991. Neuritic pathology and dementia in Alzheimer's disease. Ann. Neurol. 30:156-165.

65. Cummings, B.J., Pike, C.J., Shankle, R., and Cotman, C.W. 1996. Beta-amyloid deposition and other measures of neuropathology predict cognitive status in Alzheimer's disease. Neurobiol. Aging. 17:921-933.

66. Grober, E., et al. 1999. Memory and mental status correlates of modified Braak staging. Neurobiol. Aging. 20:573-579.

67. Mattson, M.P., Keller, J.N., and Begley, J.G. 1998. Evidence for synaptic apoptosis. Exp. Neurol. 153:35-48.

68. Mattson, M.P., Partin, J., and Begley, J.G. 1998. Amyloid beta-peptide induces apoptosis-related events in synapses and dendrites. Brain Res. 807:167-176.

69. McLaughlin, B., et al. 2003. Caspase 3 activation is essential for neuroprotection in preconditioning. Proc. Natl. Acad. Sci. U. S. A. 100:715-720.

70. McLaughlin, B. 2004. The kinder side of killer proteases: caspase activation contributes to neuroprotection and CNS remodeling. Apoptosis. 9:111-121.

71. Chung, C.W., et al. 2003. Atypical role of proximal caspase-8 in truncated Tau-indued neurite regression and neuron cell death. Neurobiol. Dis. 14:557-566.

72. Zhang, Y., McLaughlin, R., Goodyer, C., and LeBlanc, A. 2002. Selective cytotoxicity of intracellular amyloid beta peptide1-42 through p53 and Bax in cultured primary human neurons. J. Cell Biol. 156:519-529.

73. Rohn, T.T., Rissman, R.A., Head, E., and Cotman, C.W. 2002. Caspase activation in the Alzheimer's disease brain: tortuous and torturous. Drug News Perspect. 15:549-557.

74. Ferrari, A., Hoerndli, F., Baechi, T., Nitsch, R.M., and Gotz, J. 2003. beta-Amyloid induces paired helical filament-like tau filaments in tissue culture. J. Biol. Chem. 278:40162-40168.

75. Ackmann, M., Wiech, H., and Mandelkow, E. 2000. Nonsaturable binding indicates clustering of tau on the microtubule surface in a paired helical filament-like conformation. J. Biol. Chem. 275:30335-30343

76. Dawson, H.N., et al. 2001. Inhibition of neuronal maturation in primary hippocampal neurons from tau deficient mice. J. Cell Sci. 114:1179-1187.

77. Lynch, J.R., et al. 2002. Apolipoprotein E affects the central nervous system response to injury and the development of cerebral edema. Ann. Neurol. 51:113-117.

78. Petersen, R.C., et al. 1999. Mild cognitive impairment: clinical characterization and outcome. Arch. Neurol. 56:303-308.

79. Higuchi, M., et al. 2002. Transgenic mouse model of tauopathies with glial pathology and nervous system degeneration. Neuron. 35:433-446.

80. Torp, R., et al. 2000. Ultrastructural evidence of fibrillar beta-amyloid associated with neuronal membranes in behaviorally characterized aged dog brains. Neuroscience. 96:495-506. 